\title{
Adrenocortical suppression in workers employed in manufacturing synthetic glucocorticosteroids: solutions to a problem
}

\author{
R W NEWTON, ${ }^{1}$ MARGARET C K BROWNING, ${ }^{1}$ P C NICHOLSON, ${ }^{2}$ AND \\ D A E MOWAT ${ }^{2}$
}

From Ninewells Hospital and Medical School, ${ }^{1}$ Dundee, and Glaxo Operations UK Limited, ${ }^{2}$ Montrose, UK

\begin{abstract}
Earlier studies showed gross adrenocortical suppression in three workers employed in manufacturing synthetic glucocorticoids. A further nine had either recognisable abnormalities of their responses to tetracosactrin or evidence of chronic skin contact with glucocorticoids. By 1978 the mean morning cortisol concentration of 20 workers employed in active steroid processes was $235 \mathrm{nmol} / \mathrm{l} \pm 28.6(8.5 \mu \mathrm{g} / \mathrm{ml} \pm 1.04)$, which was significantly lower than the value of $428 \mathrm{nmol} / \mathrm{l}$ $\pm 37.7(15.5 \mu \mathrm{g} / \mathrm{ml} \pm 1.40)$ obtained in a control group of 19 men $(\mathrm{p}<0.005)$. The mean morning cortisol of workers employed in processing steroids believed to be physiologically inactive, 306 $\mathrm{nmol} / 1 \pm 22 \cdot 4(11 \cdot 1 \mu \mathrm{g} / \mathrm{ml} \pm 0 \cdot 81)$, was also significantly lower than this control group $(0 \cdot 01>\mathrm{p}>$ $0.005)$. Improvement of protection for workers together with operational and plant changes resulted in a significant rise in the mean morning cortisol values of workers until, in 1979, men employed in all steroid processes had concentrations that did not differ from those of a control group.
\end{abstract}

Workers employed manufacturing potent glucocorticoids are at risk of absorbing these drugs which may produce a suppression of adrenocortical function. In 1977 Farina $^{1}$ reported clinical features similar to those seen in Cushing's syndrome in nine such workers. In 1978 we reported the results obtained in 12 workers employed in the synthesis of betamethasone. ${ }^{2}$ Three had gross adrenocortical suppression, a further five had abnormal responses to tetraconsactrin, and all 12 showed evidence of chronic skin contact with glucocorticoids.

Until our previous report, the hazard of adrenocortical suppression in "steroid operatives" was not fully appreciated. Recognition of the risks resulted in regular biochemical screening of the workers and monitoring of the working environment as well as technical modifications to the manufacturing process. We describe the evolution of these changes and their practical effect in reducing the health risk to those employed in the manufacture of glucocorticoids.

\section{Plant and environmental changes}

The production of physiologically active gluco-

Received 18 March 1981

Accepted 29 May 1981 corticoids from natural raw materials containing the basic cyclopentano-perhydro-molecule is a complex multi-stage process (figs 1 and 2). The initial mixing of the raw materials takes place in enclosed reaction vessels. Intermediate and final products, in the form of a wet precipitate, then undergo the "finishing stages" of crystallisation, filtration on open pan filters, drying of the product on trays in a vacuum oven, bulk bagging of active material, and subsequent milling, granulating, and micronisation to reduce particle size. Because of difficulties in confining dust particles created during micronisation, this process has always been carried out in a purpose-built suite. Initially operators were protected by disposable overalls, gloves, and air hoods; at other less dusty finishing stages only limited protective clothing was worn.

Before a serious occupational health hazard was identified in 1977 no routine monitoring of active steroid dust levels in the environment took place. Two of the three workers showing severe adrenocortical suppression were employed in the micronising process and in 1978 operators in this area were clothed in full air suits rather than air hoods, and facilities were provided for decontaminating and storing of the suits. Further recommendations were that dust levels of active glucocorticoids at other 


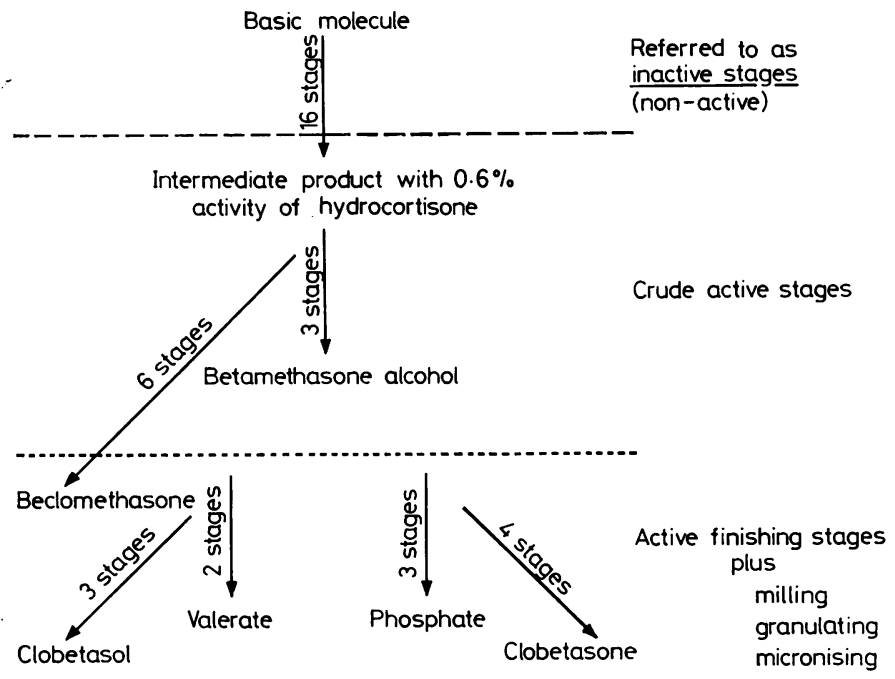

Fig 1 Reaction sequences in manufacturing synthetic glucocorticoids.

finishing stages should be measured, and that strict rotation of men from the micronising process to other work should take place to prevent continuous exposure. There was, however, some delay before these recommendations were implemented.

Regular collection of airborne dust by membrane filters and "fall plates" with subsequent analysis identified other hazardous areas. Unacceptably high concentrations of active glucocorticoids were found
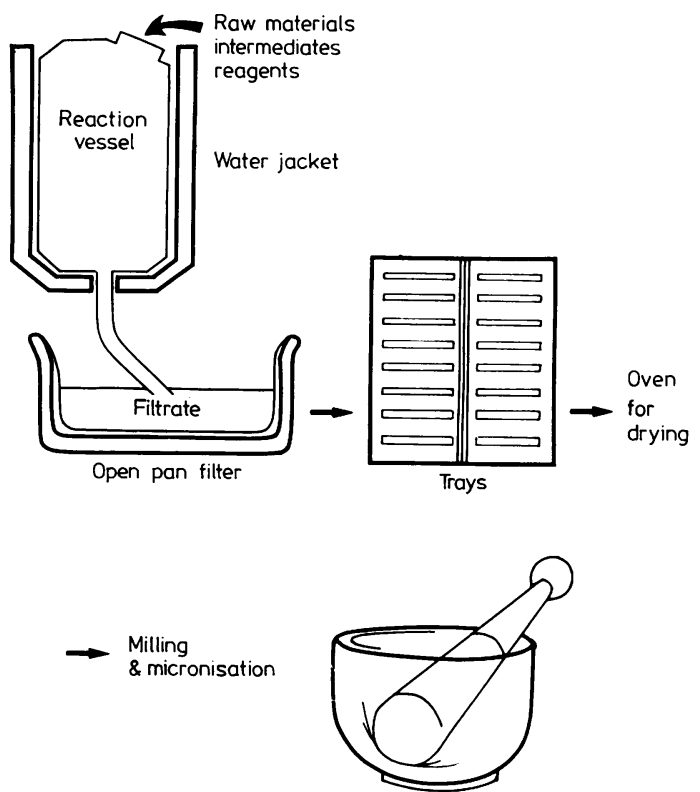

Fig 2 Diagrammatic representation of stages of manufacture of synthetic glucocorticoids. in the drying area. The wearing of air hoods instead of conventional face masks was introduced in these areas and later full air suits with decontamination and storage facilities were provided. Considerable air and plant contamination during wet processes and transient local contamination during the charging of the reacton vessels was found. Full air suit protection was introduced during charging operations, and unprotected personnel were excluded from the reaction vessel area.

The ventilation of most finishing rooms had been designed to provide slight positive pressure to prevent secondary contamination of the finished product by impurities. Dust monitoring showed resultant contamination of adjoining changing rooms and plant areas. Extensive modifications to the ventilation and extraction systems included replacement of laminar air flow barriers with solid walls, reversal of air pressures, and the inclusion of air locks.

The finding of active steroid levels on the exterior surface of air suits highlighted the extreme difficulties of decontamination due to the insolubility of most glucocorticoids in water. Measurable levels of active steroid within these suits may have lead in some instances to a "steroid occlusive dressing" effect. More efficient decontamination of air suits was accomplished by using detergents and a two-man operation. These modifications were completed by mid 1979.

\section{Clinical and biochemical screening}

In 1977 all workers in areas then recognised as being hazardous had tetracosactin tests carried out. Previously no screening of adrenocortical function 
had taken place. Subsequently operatives were examined clinically at six-monthly intervals, and blood was taken at 9 am for serum cortisol estimation. Where this was $140 \mathrm{mnol} / \mathrm{l}(5 \mu \mathrm{g} / \mathrm{ml})$ or less a tetracosactin test was performed.

Continuing evidence of adrenocortical suppression in late 1978 led to more regular screening of serum cortisol concentrations in all the workers employed in the steroid manufacturing process. Subjects were screened at the end of each two-month period in the active steroid processing area and again at the end of a two-month period when they were believed not to have been exposed. The results obtained were compared with the morning plasma cortisol values in a control group of 19 workers who were assessed at the beginning of employment with the pharmaceutical company and therefore before possible exposure to glucocorticoid manufacturing processes. In 1979 the criteria for assessing adrenocortical reserve were changed to include men in whom the morning cortisol value was between $140 \mathrm{nmol} / 1(5 \mu \mathrm{g} / \mathrm{ml})$ and $280 \mathrm{nmol} / \mathrm{l}(10 \mu \mathrm{g} / \mathrm{ml})$ on two samples withdrawn within seven days or in whom the cortisol values despite remaining within the normal range showed a fall on three consecutive occasions.

\section{Results}

Work in 1977 had shown adrenocortical suppression in three workers employed in manufacturing potent synthetic glucocorticoids. Clinical evidence of chronic skin exposure to steroids was found in a further nine. By 1978 a system of regular rotation of workers to processes believed to include physiologically inactive steroid had been instituted, and several technical modifications to the plant were undertaken. At that time the mean morning serum cortisol value ( \pm SE of mean) in 20 workers employed in active steroid processes was $235 \mathrm{nmol} / 1$ $\pm 28.6(8.5 \mu \mathrm{g} / \mathrm{ml} \pm 1.04)$. This was significantly lower than the mean of $428 \mathrm{nmol} / \mathrm{l} \pm 37 \cdot 7(15.5$ $\mu \mathrm{g} / \mathrm{ml} \pm 1.40$ ) obtained in a control group of 19 men before employment by the pharmaceutical company $(p<0.005)$. In addition it was significantly less than in eight workers believed to be working in processes using steroids considered to be physiologically inactive $(306 \mathrm{nmol} / \mathrm{l} \pm 22.4(11 \cdot 1 \mu \mathrm{g} / \mathrm{ml} \pm$ $0.81) 0.05>p>0.025)$. Interestingly, however, this value was also significantly lower than the mean morning cortisol value obtained in the control group $(0.01>\mathrm{p}>0.005)$.

The results of mean morning serum cortisol estimations for three-month monitoring periods are shown in fig 3. Over a two-year period there was a gradual rise in the morning cortisol concentrations of workers known to be employed in "active processes."

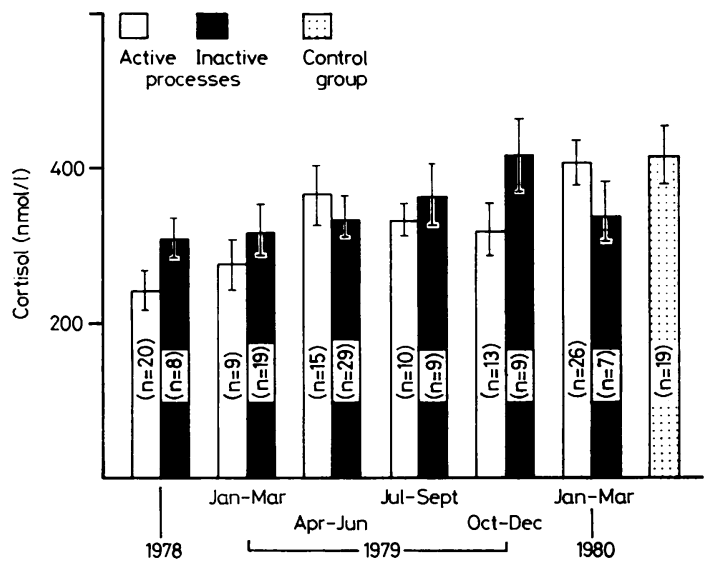

Fig 3 Mean morning plasma cortisol values of workers employed in manufacturing synthetic glucocorticoids during consecutive three-month monitoring periods.

During the last two three-month periods there was no significant difference from control values reflecting the technical modifications, environmental improvements, and better operator protection. As late as June 1979 workers operating in processes believed to include non-physiologically active steroids had a mean morning cortisol value that was significantly lower than the control group. Reappraisal of these process areas and reassessment of the physiological activity of a number of steroid intermediates and institution of appropriate precautions resulted in a rise in cortisol concentrations in these workers until during the last three consecutive monitoring periods there was no significant difference from cortisol concentrations obtained in unexposed workers.

\section{Discussion}

Figure 3 shows significant differences in adrenocortical function between workers exposed to the physiological active steroids and control subjects in 1978. Work in 1977 had highlighted exposure to the glucocorticoids manufacturing process as a serious health hazard leading to adrenocortical suppression in some workers, but the importance of the initial recommendations was not fully appreciated. Environmental monitoring for glucocorticoids and adherence to rotation programmes were neglected. The effects of exposure to physiologically active steroids are reflected in the cortisol concentrations recorded during 1978. Discussions between medical and management personnel led to more regular monitoring of the environment and the operators. Further changes to the industrial processes and more efficient air suit protection resulted in a striking 
reduction in the level of exposure to glucocorticoid reflected by mean morning cortisol values. Surprisingly, some men working in processes believed to include non-physiologically active intermediates had evidence of adrenocortical suppression. Analysis of environmental dust and surface swabs showed considerable cross-contamination of these areas from the physiologically active glucocorticoid process areas. Where indicated, the industrial processes and procedures were modified and increased protection was given to the operators. The need for re-educating and retraining operators became apparent.

The importance of careful environmental monitoring and the close co-operation between management and medical personnel in devising a system of occupational health care for these steroid workers cannot be overestimated.

The value of regular environmental monitoring was established. Closer scrutiny of the physiological activity of certain steroid intermediates showed some activity in two previously believed to be inactive. Increased protection of operators handling these compounds led to improvement in the mean morning serum cortisol value.

Determining a safe environmental working level for pharmaceutical compounds is always difficult but becomes increasingly so when the drug is highly active and can be absorbed via the lungs, gastrointestinal tract, and percutaneously. Even with the most up-to-date chemical manufacture complete freedom from exposure cannot be guaranteed. For certain operations complete protection of the operator will always be necessary together with continued environmental and biochemical monitoring.

\section{Conclusion}

The recognition of adrenocortical suppression in workers employed in manufacturing potent glucocorticoids and the steps that have been taken to reduce the hazard have emphasised the value of close co-operation between medical and industrial personnel in achieving a solution. Careful environmental monitoring, regular rotation of operatives away from steroid processes, and an orderly system of biochemical monitoring of operatives have had a considerable effect in reducing adrenocortical suppression in these workers. Unless fully contained processes are in use steroid workers elsewhere must be presumed to be exposed to a similar risk. Lack of symptoms and overt illness should not be accepted as evidence of normal adrenocortical function in those employed in manufacturing glucocorticoids.

\section{References}

${ }^{1}$ Farina G, De Micheli P, Basso P, Castelli PP, Locati G, Secchi GC. First report on an exogenous hypercortisolism syndrome in workers engaged in the manufacture of steroids. Med Lav 1977;68:167-77.

${ }^{2}$ Newton RW, Browning CK, Iqbal J, Piercy N, Adamson DG. Adrenocortical suppression in workers manufacturing synthetic glucocorticoids. Br Med J 1978;i:73-4. 